\title{
Codifying an approach to remote practicals
}

\author{
Garrard, A. ${ }^{1}$, Bates, J., Beck, S.B.M and Funnell, A. C.
}

All authors are from: The Department of Multidisciplinary Engineering Education, The University of Sheffield, Faculty of Engineering, The Diamond, Sheffield, S3 7RD, UK

Keywords: practical engineering education; remote practicals; blended learning.

\begin{abstract}
Multidisciplinary Engineering Education (MEE) at the University of Sheffield is dedicated to delivering, at scale, practical teaching to students in the Faculty of Engineering. The COVID-19 pandemic initiated the sudden suspension of face to face teaching required MEE to translate over 600 in-lab practicals to a remote delivery format. With little opportunity to coordinate, academic staff independently adopted a variety of tactics to ensure practical learning outcomes were maintained. Following the reactive response, a proactive reflection was conducted and six categories of tactics for remote practicals have been established. These categories are Provide digital artefacts; Simulated practicals; Synchronous remote participation; Asynchronous participation by proxy; Perform procedure in alternative environment; Remote staff support. The advantages and drawbacks of each of these categories is discussed and it is suggested which tactics are appropriate for particular learning outcomes or operational and environmental outcomes of equivalent in-lab practicals. Further work to comprehensively align outcomes to tactics is proposed and lasting benefit from the analysis can be realized by adopting a principle of Remote Enhanced Practicals.
\end{abstract}

\section{Introduction}

Multidisciplinary Engineering Education (MEE) at The University of Sheffield is a department dedicated to the delivery of practical teaching for all students in the Faculty of Engineering. This teaching includes laboratory and workshop activities for over 4,500 undergraduate students studying one of a range of 10 major undergraduate courses, such as Civil, Electrical or Chemical Engineering, and over 2,000 taught postgraduate students. The volume of practical teaching delivered by MEE and the collaborative nature of working across multiple engineering departments justifies a generous resource allocation, through the combination of modest contributions from each cooperating department (Garrard and Beck 2018). Centralising the practical teaching also allows efficiency in the use of the resources due to economies of scale. For example, MEE invests time and effort developing effective learning and teaching infrastructure for the whole department, such as web and mobile applications, which can support academic staff innovation through minimising time spent on administrative tasks.

\footnotetext{
${ }^{1}$ Corresponding Author: a.garrard@sheffield.ac.uk
} 
MEE employs a team of 15 academic staff and runs 8 large capacity and 5 specialist laboratories, accommodating multiple copies of equipment so all students in the sessions can perform the same experiment at the same time. This investment in large-scale resources facilitates two pedagogical advantages:

1. Focus on teaching excellence in practical engineering education.

2. Practical activities can be taught to large cohorts in few timetabled sessions.

In a traditional academic workload allocation model, academic staff would typically be responsible for delivering many forms of teaching on a small number of courses (or modules). For example, they may be responsible for the lectures, tutorials, lab classes, coursework and exam assessments. MEE's novel teaching model is a volte-face to this approach and staff deliver only a specific form of teaching, i.e. the practical element, to a large number of courses (or modules). Dedication to practical engineering education allows academic staff focus, space and experience to consider the pedagogy of this type of teaching in significant depth and develop expertise in the field.

In a more tightly resource constrained environment, where only limited sets of equipment can be justified, timetabling pressures and student numbers means the date on which a student performs a particular activity may not be known with precision. Conversely, MEE can schedule practical activities for all students in a specific week to, for example, coincide with associated theoretical material delivered in lectures. Therefore all students on a particular programme of study follow a predefined sequence of practical activities.

The result of these two pedagogical advantages of the MEE teaching model is carefully considered learning outcomes for each activity. As the sequence of student's activities is defined, staff can focus on ensuring the learning from practicals is conceptualised and articulated both internally and with students. Learning outcomes can be mapped and designed to ensure progression in rigour and student expectation from one activity to the next.

On Friday 13th March 2020 the University of Sheffield announced a suspension of face to face teaching, including in-lab practicals, to commence on the following Monday. For classroom based teaching, such as lectures and tutorials, established solutions, both pedagogical (e.g. flipped learning) and technological (e.g. Blackboard Collaborate, Zoom), exist and have been reasonably well adopted across the sector. For practical education, which typically involves activities focused around a tangible artifact, tool or piece of equipment, the range of options for transition to remote delivery have not been as well explored.

As was the case with most other HE institutions, MEE adopted a reactive approach to the suspension of face to face teaching due to the short timescale, aiming to minimise disruption to students as much as possible (Nordmann et el 2020). Academic staff were instructed to develop remote practicals to replace the scheduled in-lab practicals using the methods most 
appropriate for their discipline with the important condition that all explicit learning outcomes were met (AHEP 3, 2020).

Coalescence of all practical teaching into a single department provided a robust framework from which academic staff could build remote practicals. This is primarily due to staff workload being dedicated to practical engineering education undistracted by complex issues around translating other forms of teaching, such as examinations, into remote delivery. In addition, organizational aspects of managing and communicating (internally and with other departments) the transition of practical teaching from the original, in-lab activities was facilitated by utilizing existing learning and teaching infrastructure. Following a planned process (Bangert et al. 2020), MEE converted over 600 scheduled sessions to be delivered as remote practicals.

Following the reactive process of converting the scheduled activities to remote practicals, MEE entered a proactive process of reflecting on the variety of teaching methods adopted by the team of academic staff. The work presented here aims to codify these methods into categories of tactics for delivering remote practicals and considers under what scenarios each may be deployed in order to meet learning learning outcomes associated with practical teaching. Aspects of in-lab practical teaching beyond the explicit learning outcomes, such as non-verbal feedback obtained from students making enquiries, are also considered, as these may be lost during remote delivery.

The overall objective of the work presented here is to rigorously identify what learning outcomes and aspects of in-lab activities can be effectively translated into remote practicals and what tactics may be adopted to achieve this transition. Equally critical is an analysis of the detriment of employing the categories of tactics for remote practicals and what learning outcomes and aspects of in-lab activities cannot effectively be translated away from an in-lab format.

It is anticipated that the reflective analysis conducted here will have a lasting impact beyond the current situation imposed by Covid-19. Though careful consideration of the learning objectives and the teaching methods employed when remote teaching was mandatory, best practice can be established and applied to deliver Remote Enhanced Practicals (REP) when conventional teaching is restored. This may be of particular importance if working practice guidelines limit the amount of contact time students have with staff and equipment, as this work could provide principles for prioritization.

\section{Learning outcomes of practicals}

In order to ensure that an activity is appropriately designed to meet specific learning outcomes, those learning outcomes need to be determined by the teacher and articulated to the students. When redeveloping an existing in-lab activity for remote delivery it is important 
to ensure that the teaching methods adopted are capable of achieving the same learning outcomes (Herrera et al, 2006).

Learning outcomes are inherently nested with increasing levels of granularity from programme level to activity specific. For example, a programme level learning outcome may be to understand and apply experimental methods to solve engineering problems. This learning outcome could apply to all practical activities undertaken by a student and encapsulates the combined learning from a diet of individual activities, each contributing a fraction to the broader goal. For example, an Electrical Engineer may be required to learn the purpose and function of an oscilloscope, which would be both an activity specific learning outcome and a component of the programme level learning outcome.

A framework for practical learning outcomes has been developed by MEE to identify how activity and session level learning outcomes contribute to programme level learning outcomes associated with practical engineering. This framework ensures there is common language between MEE staff and teaching staff in other departments to describe how learning outcomes relate to one another. This is a particularly powerful tool for programmes delivered across multiple university departments, for example, although all practical activities for aerospace engineers are delivered by MEE, other teaching is provided by the electrical, mechanical and civil engineering departments, among others. Students and staff can identify and reflect on how individual components of their learning contribute to their overall programme. It allows mapping to ensure coverage of a variety of aspects of practical engineering and identify areas where

Learning Outcomes serve two tasks. They provide a threshold above which compliance with the outcome can be determined and a mechanism to differentiate between students of different standards. It is also good practice, and particularly pertinent to practical teaching, to adopt spiral learning (Harden, 1999) and allow students multiple exposures to learning opportunities with increasing level of expectation. For this reason, the framework adopted by MEE is staged, with progressively increasing levels of complexity and rigour for each of learning outcomes. These are referred to as our Practical Engineering Learning Outcome Level Descriptors (LOLD).

Categories of practical learning outcomes are discussed here as the objective of this work is to determine which can and cannot be effectively delivered remotely. The Practical Engineering Learning Outcome Level Descriptors (LOLD) provide a comprehensive list to align to tactics for remote practicals. In this framework, five overarching reasons have been identified for including practical teaching within an engineering programme:

1. Demonstrate a concept: Illuminating a theoretical concept manifest in the real world through experimentation or application.

2. Integrate practical and theoretical learning. Integrating prior knowledge into application and using results of experimentation to enhance theoretical understanding. 
Using the results of experimentation to update an appreciation of how closely theoretical concepts fit with the real world.

3. Solve engineering problems. Applying practical skills to situations where the results are not known. Understanding where experimentation can be used in preference or in conjunction with theory or simulation.

4. Develop a range of experimental competencies: Understand and apply the skills necessary to operate effectively in a workshop or laboratory environment.

5. An alternative to classroom based work: Conducting practical work should be an enjoyable part of the curriculum, providing inspiration and texture to the methods employed across the programme. Practicals accommodate students with a predisposition for a hands on learning style.

Learning outcome 4 is subdivided into 7 experimental competencies.

1. Designing experiments: Understanding and applying the principles of the scientific method and experimental design.

2. Production skills. Selection of the methods, tools and production required to realise a product and understanding of associated constraints.

3. Managing risk. Recognising where risk occurs in practical activities and being able to assess and manage this in a laboratory or workshop (Johnson et al, 2016).

4. Handling error and uncertainty. Methods to understand, estimate, record, process and present error, accuracy and uncertainty associated with the experimental process.

5. Keeping an experimental record. Recording methods, observations and data during an experiment in a clear and professional way.

6. Practical skills: Practical skills are those that enable students to understand and apply the techniques and experimental equipment of their discipline.

7. Communicating experimental findings: Communicating results of experimental work, in a variety of ways to a variety of audiences.

All labsheets used by MEE explicitly state the activity's learning outcomes and the associated level descriptor category on the cover of the lab sheet It is therefore clear what type of learning is required when the in-lab practical is translated to a remote practical.

It is important to appreciate that while executing an in-lab practical, a significantly larger amount of learning will occur than the explicitly stated learning outcomes. For example, if a laboratory activity is conducted specifically to observe the outcome of an experiment (concept) and communicate the findings, a process of determining a data capture strategy (experimental design), operating the equipment (practical skills) and keeping the results in an experimental record will need to be applied. To differentiate between learning outcomes that are deliberately chosen as part of curriculum design, and those that are a latent and unavoidable part of conducting practical work, the following terms are used: 
Explicit learning outcomes: Those that are communicated to the students as the focus of the activity, then taught, practised by students and finally assessed.

Implicit learning outcomes: Learning that occurs as a result of performing practical activities, but not specifically communicated to the student or assessed. Discussions around implicit learning can occur if promoted by students.

Implicit learning outcomes have been touched upon in the literature, but principally from the perspective of how to make them explicit (Hyytinen and Löfström, 2017). In a practical context, awareness of the implicit learning outcomes should be maintained by staff, for mapping and audit purposes. However, it is important not to dilute the focus of a taught session by communicating these supplementary outcomes to students.

In a reactive scenario when rapidly implementing change to teaching delivery methods, adherence to the existing explicit learning outcomes is necessary to ensure veracity of the programme. The resources to also deliver the implicit learning outcomes may not be available. This guided our approach to transitioning to remote practicals, by making it mandatory to teach to the same learning outcomes as originally planned, while producing an effective operating environment to contribute to implicit learning.

\section{Operational and environmental outcomes of in-lab practicals}

This section addresses the incidental outcomes which students can achieve during in-lab practical sessions. We describe some of these outcomes as "operational" because they are often the result of pragmatic choices made by the teaching team to efficiently deliver in-lab sessions, and could be delivered pre-, in- or post- laboratory sessions. We also consider "environmental" outcomes that are strictly correlated to working in laboratory environments and the tasks performed in specialist spaces.

The operational and environmental outcomes (OEOs) are not notified to students as explicit learning outcomes, however teachers and curriculum designers need to be aware of all aspects of in-lab sessions to take advantage of additional developmental opportunities for students. It is also notable that these outcomes are based not only on operation and environment, but also on individual students' personality and implicit knowledge.

Some OEOs can be described by the concepts of Incidental Learning, where learning is unintentional or unplanned from other activities (Kerka, 2000), and Discovery Learning, when the learner is not provided with the target information or conceptual understanding and must find it independently and with only the provided materials (Alfieri et al, 2011). 
We have looked at different operational and environmental aspects of the lab session and how they can influence the student learning.

\subsection{Team working:}

Most lab activities for taught undergraduate students require the students to work in teams rather than individually. The teams must collaborate to perform a range of tasks including sharing equipment for data gathering up to a more complex journey of experimental planning, requiring the collation, communication and evaluation of information.

It is ideal to develop a 'social capital' connection between the team members. 'Social capital' as a concept has been extensively analysed in a public policy environment (Lewis, 2009), where the definition of 'social capital' is 'connections among individuals - social networks and the norms of reciprocity and trustworthiness that arise from them' (Putnam, 2000). A cohesive and trustworthy team in the university environment can achieve a sense of a community of peers, where members can take a different role as leader, negotiator, organiser and possibly teacher. An in-lab collaborative environment is ideal for developing such connectivity, which students can then take into other areas of their studies.

\subsection{Pre-, post- and in-lab activity design:}

Pre-lab and post-lab activities are an essential part in the lab's learning explicit and implicit outcomes. The pre-lab activities allow the students to learn about the theory, Health and Safety implications and possible calculations needed during the session. In MEE, students must submit a piece of pre-lab work before attending the lab session. The post-lab activities reaffirm what the students have learned during the lab session; they must complete a post-lab task, often in the form of an online quiz or report, before a deadline. For many lab activities the students can access extra reading material or video, which can explain in more details the equipment which will be used in order to help them to plan scientific investigations, or are given extra maths tuition which should help them to analyse and interpret data.

\subsection{Extensions to in-lab activities:}

Although staff design in-lab activities to fill structured, timetabled sessions, students are generally able to build upon the provided exercises and make further use of the equipment to explore around the subject. This may include recognising erroneous data and repeating measurements, or attempting to perform the same experiment with alternative equipment to compare and contrast the results achieved. The most engaged students may be keen to perform another experiment entirely, for their own self-development beyond the course material. 
When transferring to remote practical activities, if it is desired for students to be able to freely continue their investigations, the structure must be sufficiently flexible to allow students to explore beyond the prescribed tasks. This is more achievable with some methods than others, for example, videos and stock datasets do not easily allow room for further exploration, while simulations can generally be extended in a free-form manner.

\subsection{Real time cause and effect:}

One of the explicit learning outcome categories defined in section 2 is Practical Skills, where the student must understand and apply the techniques and experimental equipment of their discipline. A more subtle outcome arises when the students are placed in front of equipment where they have to learn to assimilate simultaneously the macro and micro aspects of the full experimental system and focus rapidly on the system inputs and outputs. This incorporates the psychomotor skills of using technical equipment alongside interpreting the cause and effect of interacting with dynamic systems.

The notion of real time cause and effect also includes the students learning from failure and troubleshooting what went wrong, including by readjusting the equipment settings or debugging a software program. The pressure of a limited amount of allocated time with the experimental equipment is also an influential factor, requiring students to take account of the time required for experimental work and make judgements on the highest quality measurements and investigations that can be performed in the time available.

\subsection{Feedback in lab:}

Different forms of feedback (quantitative and qualitative) can be given to students. The students are mostly interested in quantitative feedback (final mark or pass/fail) however; the final mark may not always help the students directly with their learning. The qualitative feedback can be formal or informal and in the lab environment, the students will receive an informal feedback. In MEE, students will generally ask for advice and feedback from a Graduate Teaching Assistant (GTA) instead of academic staff during the lab session, due to the class size. In the latter case, the feedback can be provided by an open discussion, where the student may be more inclined to ask questions, discuss their doubts and enquire why something is not working.

In-lab feedback allows the student to receive constructive advice on their progress very quickly, with dedicated staff in the room to assist with the specific activity being undertaken at the time.

We need to be aware of all of these attributes to ensure they are not lost when moving to remote practicals, or at least be sure that their loss is not critical to the explicit learning outcomes. Teamwork can possibly still be achieved using online calling technologies and shared online workspaces, despite the challenges of remote communication between the team 
members. Additional, optional lab activities could also be provided to students just as they were always meant to be delivered, outside of the scheduled lab session. Probably the most challenging outcome will be real time cause and effect, unless we will be able to provide synchronous remote equipment manipulation. Feedback in the lab could be another challenging outcome when moving to remote practicals.

\section{Tactics for remote practicals}

Following the sudden announcement of the suspension of all in-lab practicals, MEE staff began the process of migrating over 600 remaining timetabled sessions to remote practicals. The reactive response involved staff members individually identifying methodologies to port their remaining scheduled practical activities to a remote format. This catalogue provides a sufficient collection of different examples of approaches to converting in-lab to remote practicals for analysis. A comprehensive review was conducted and found that all of the approaches employed could be codified into 6 categories of tactics for delivering remote practicals:

1. Provide digital artefacts

2. Simulated practicals

3. Synchronous remote participation

4. Asynchronous participation by proxy

5. Perform procedure in alternative environment

6. Remote staff support

When considering a tactic to adopt, consideration should be made to the importance of synchronicity. Synchronous delivery has the advantage of creating a community of peers and helps students structure their learning, whereas asynchronous delivery can create flexibility for both staff and students to plan their work and overcomes issues of varying time zones. All teaching methods are either a) inherently synchronous or b) can be designed to be synchronous or asynchronous. Any asynchronous activity can be made synchronous by imposing specific time constraints, for instance limiting access to pre-recorded videos. In each of the tactics discussed, the synchronicity will be stated.

\subsection{Category 1: Provide digital artefacts}

This method provides the same visual and/or aural stimuli or simply the raw information that students would have gained from an in-lab practical, by digitising and distributing these data. Examples of this would be to create still images or video of experimental observations or data to represent the readings that would have been collected from instrumentation during the experiment, either stock data from the real equipment or data that is illustrative of the system. 
Providing digital artefacts allows students to see the operation of equipment and process data to infer findings. An advantage of the asynchronous distribution of the content allows students to repeat the process many times to reinforce the learning, and the method is easily scalable to large cohorts, although the production of good quality digital teaching material can be time consuming. Teaching staff provide several media clips or datasets to show the variation of outcomes over multiple, ostensibly identical, repetitions of procedure.

A significant drawback to this approach is it limits the opportunity for students to make decisions, such as what data to collect and what equipment to focus upon. It is also difficult to capture the essence of a real time cause and effect response from physical systems.

\subsection{Category 2: Simulated Practicals}

The limitation of being able to make certain decisions about how to execute an experiment can be overcome by creating simulations of a physical system (Balamuralithara and Woods, 2009). For example, the provision of stock data eliminates the need to develop a data capture strategy by pre-selecting appropriate values of independent variables. A simulation could generate the dependent variable based on any input independent variable.

Simulations can be built with a range of sophistication. To generate numerical data, a well written spreadsheet can be produced. Deliberately introduced random errors can be programmed into the output of the spreadsheet in order to replicate data collected from real world equipment, providing the potential for students to rapidly repeat experiments multiple times to see a distribution in results.

The use of 3D computer aided design (CAD) models visible on screens or fully immersive VR experiences can replicate the ability to investigate and probe physical equipment. Within MEE many pieces of experimental apparatus are designed and built in-house, providing ready access to share CAD with students though the virtual learning environment (Bangert 2020).

Practical Engineering Education simulations can be built in-house or purchased from suppliers. In either case, with increasing level of sophistication comes increased cost, be it financial or staff time. One of MEE's specialist facilities is a pharmaceutical pilot plant for the large scale manufacture of pills. A "digital twin" of the facility exists, replicating all systems in a simulated environment with identical control interfaces, allowing students remote access to the facility. In addition to the advantage of more flexible asynchronous access, the digital version of the equipment allows the exploration of non-physical or impractical (for example, dangerous) operation to determine outcomes that would not be permitted in the laboratory.

Simulated experiments have the significant drawback of lacking "realness" and the tactile enjoyment of playing with tangible artefacts. There are also limits on the amount of exploration available for students, given that they can only operate within the predetermined 
structure of the functions of the simulation. The "what if" scenarios are constrained by the imagination of the developer and the budget of their construction.

\subsection{Category 3: Synchronous remote participation}

Remote access to in-lab equipment can be achieved in one of two ways. Firstly, through live demonstration, where a member of staff hosts an interactive session within the experimental facility and broadcasts this to students. Methods for capturing and distributing video, audio and/or data are required. Consideration also needs to be given to managing triage of queries and questions, and queuing of students wishing to communicate with staff or the whole group. However, technology for these tasks is well established and available at most HE institutes for staff use.

An alternative strategy for delivering synchronous remote participation is the use of telemetry for actuation of equipment and measurement of the result. Depending on the amount of physical movement, the ability to operate the equipment remotely may be provided within its normal capability, or it may be necessary to retrofit components to create this functionality. The increasing prevalence of IoT devices opens opportunities to control and sense using relatively inexpensive products. However, technological solutions for scheduling and networking will be required, to allow students to access the control of the equipment at specific times. This could mean that limited amounts of equipment are required, as they can be accessed over an extended time period, rather than a fixed lab session.

Inherently synchronous delivery has all the advantages associated with creating an event attended by a community of peers learning together. Staff and teaching assistance can be available "live" to provide instantaneous feedback as required. Synchronicity also allows the retention of systems put in place for in-lab activities, to imply parity with remote equivalents. For example, MEE required the completion of an online, pre-lab activity before students could commence any in-lab activity, to ensure students were safe and prepared. While these requirements can be placed on asynchronous activity, the concept of tasks to complete before the event is, to a certain extent, diluted with an ill-defined start time.

\subsection{Category 4: Asynchronous participation by proxy}

Professional process for executing practical work is to plan, in advance, the procedure to adopt. Asynchronous participation by proxy involves the work being designed by the student and executed by another person, typically a member of staff or teaching assistant, and the results reported back. The process could involve discussion between the student and person following the procedure prior to execution, to reflect on the design and documentation of the proposed process.

Experimental design learning outcomes could be fulfilled using this method. It may also be pertinent to fulfilling learning outcomes associated with fabrication, for remote access to 
tools and workshops. This approach closely replicates engineering in industry, where designers submit drawings to be manufactured and test protocols to be followed. There is an opportunity for students to acknowledge the experience of operators and identify the value of using other people's expertise as a resource.

This method provides freedom to work outside structured teaching formats that other tactics for remote practicals may constrain. Therefore it can be useful for more open ended learning tasks. However, there are significant implications for staff time resources.

\subsection{Category 5: Perform procedure in alternative environment}

It may be possible to perform practical tasks outside of laboratory spaces. If explicit learning outcomes relate to the development of experimental competencies this learning can be delivered without the need to access specialist equipment. For example, the ability to keep an experimental record or quantify experimental uncertainty, could be achieved following a cooking recipe. Conducting practical tasks with equipment that students are expected to own presents challenges with the control of the outcomes and equity of access within a chort. An alternative is to provide "take home kits" that students are able to design, operate and analyse away from university space (Patterson, 2019). This method will have cost implications, which scale with cohort size, but increases the consistency of student experience.

While the range of physical concepts students will be able to investigate is limited, and the precision of the instrumentation may be poor compared to that found in a laboratory, these options provide "real" results and can be fun, engaging and inspiring. Health and safety, and risk need to be carefully considered when employing this tactic.

\subsection{Category 6: Remote staff support}

The final category is the collection of tactics that can be employed to provide remote access to the function of teaching staff, that would normally be available during in-lab practicals. Experienced practical engineering educators:

- Ensure sessions run to plan and maintain students' concentration and momentum.

- Monitor situations requiring attention to dynamically adjust methods, including safety concerns.

- Provide assistance when required to ensure all students are able to deliver the final results of the tasks or extend learning beyond the structured activity.

- Gather formal and non formal feedback about the student experience.

A number of tactics can be deployed to replicate staff involvement in a remote format to provide these functions. Digital video conference tools can be used when practicals are delivered in a synchronous format (or an asynchronous delivery format made synchronous when bound by predefined start and end times). Session planning can be replicated remotely 
with clearly labeled milestones. Milestones can be facilitated by virtual learning environments though the use of "adaptive release", i.e. downstream parts of an activity are only accessible once an upstream task has met a certain set of conditions. The processing of numerical data can be performed remotely with "check my answer" spreadsheets that compare the steps in a student's work to the "correct" answer and allow them to debug where mistakes have occurred. Such spreadsheets can emulate a teaching assistant working through calculation steps to provide bespoke feedback, rather than simply giving away "correct" answers. Finally, feedback from sessions can be gathered using existing online tools such as surveys and polls.

\section{Discussion and Conclusions}

The learning outcomes for practical engineering education and the operational and environmental outcomes of delivering in-lab practical have been presented. When considering tactics for delivering remote practicals it is critical to determine which of these outcomes need to be fulfilled. All teaching activities are vehicles for achieving learning outcomes and when translating an existing in-lab practical to a remote practical it may be that the activity can change significantly while maintaining the learning outcomes. Therefore it is critical that the contribution of activities' learning outcomes to the overall programme is made explicit. Six categories of tactic for delivering remote practicals have been distilled from a large set of case studies from MEE. Their advantages and disadvantages have been discussed and examples of what learning, operations and environmental outcomes each may be suitable. It is proposed that further work of comprehensively aligning outcomes to tactics.

\subsection{Further work}

Further work to be undertaken is to develop a "playbook", from which educators could get inspiration on methods for achieving specific practical learning outcomes. In addition this analysis will clearly illustrate what is necessarily lost from exclusively remote delivery of practical engineering education and hence reinforce the value of in-lab activities.

Furthermore, benefits can be gained for in-lab practicals by considering the tactics for remote practicals. With a rigorous model of the purposes for conducting in-lab practicals and what is effectively and ineffectively taught using remote methods, the limited contact time with students can be used for maximum effect. By blending tasks suited to face to face in-lab practicals with the tasks that are suited to remote teaching, a hybrid model of Remote Enhanced Practicals (REPs) can be delivered, based on sound pedagogical reasoning.

Remote enhanced practicals are beneficial due to the advantages of scale and efficiency, but their adoption may be prescient for an education sector where social distancing forces resource constrained scenarios and limits to in-lab teaching time. 


\section{References}

AHEP 3, The Accreditation of Higher Education Programmes: Third edition', https://www.engc.org.uk/engcdocuments/internet/Website/Accreditation $\% 20$ of $\% 20$ Higher $\%$ 20Education $\% 20$ Programmes $\% 20$ third\%20edition\%20(1).pdf (Accessed: $27^{\text {th }}$ June 2020)

Alfieri, L., Brooks, P. J., Aldrich, N. J., and Tenenbaum, H. R. (2011). Does discovery-based instruction enhance learning? Journal of Educational Psychology, 103(1), 1-18. https://doi.org/10.1037/a0021017

Balamuralithara, B. and Woods, P.C. (2009), Virtual laboratories in engineering education: The simulation lab and remote lab. Comput. Appl. Eng. Educ., 17: 108-118. doi:10.1002/cae.20186

Bangert, K. (2020) 'Experimental Design for Practical Engineering Education', Practical Engineering Education, 4 June. Available at:

https:/practicalengineeringeducation.blogspot.com/2020/06/experimental-design-for-practica 1.html (Accessed: 9th July 2020).

Bangert, K., Bates, Beck, S.B.M., J., Bishop, Z.K., Di Benedetti, M., Fullwood, J., Funnell, A., Garrard, A., Hayes, S.A., Howard, T., Johnson, C., Jones, M.R., Lazari, P., Mukherjee, J., Omar, C.S., Taylor, B., Thorley, R.M., Williams, G.L. and Woolley, R. (2020). 'Remote Practicals in the Time of Coronavirus, a Multidisciplinary Approach', 3 June. Available at: https://engrxiv.org/d7rkg/ doi:10.31224/osf.io/d7rkg (Accessed: 9th July 2020)

Garrard, A and Beck, S.B.M. (2018) Pedagogical and cost advantages of a multidisciplinary approach to delivering practical teaching, The Interdisciplinary Future of Engineering Education Breaking Through Boundaries in Teaching and Learning, ed Kapranos. P., Routledge.

Harden, R.M., (1999) What is a spiral curriculum?, Medical Teacher, 21:2, 141-143, DOI: $10.1080 / 01421599979752$

Herrera O.A., Alves G.R., Fuller D and Aldunate R.G. (2006) Remote Lab Experiments: Opening Possibilities for Distance Learning in Engineering Fields. In: Kumar D., Turner J. (eds) Education for the 21 st Century - Impact of ICT and Digital Resources. IFIP WCC TC3, vol 210. Springer, Boston, MA

Hyytinen, H.and Löfström, E. (2017) Reactively, Proactively, Implicitly, Explicitly? Academics' Pedagogical Conceptions of how to Promote Research Ethics and Integrity. J Acad Ethics 15, 23-41. https://doi.org/10.1007/s10805-016-9271-9 
Johnson, C, Bates, J., McLaughlin, K., Mason, S., A and Dean, J.S., (2016) A sweeter way of teaching health and safety, Physics Education, DOI: 10.1088/0031-9120/51/5/053006

Kerka, S. (2000). "Incidental Learning”, ERIC Trends and Issues Alert No. 18. :

https://files.eric.ed.gov/fulltext/ED446234.pdf (Accessed: $10^{\text {th }}$ June 2020)

Lewis, J., (2009) Connecting and Cooperating : Social Capital and Public Policy, University of NSW Press, ProQuest Ebook Central, http://ebookcentral.proquest.com/lib/sheffield/detail.action?docID=533820

Nordmann, E., Horlin, C., Hutchison, J., Murray, J., Robson, L., Seery, M., and MacKay, J. R. D., (2020). 10 simple rules for supporting a temporary online pivot in higher education. https://doi.org/10.31234/osf.io/qdh25 (Accessed: $4^{\text {th }}$ June 2020)

Patterson, E. A. (2019). Using everyday engineering examples to engage learners on a massive open online course. International Journal of Mechanical Engineering Education. https://doi.org/10.1177/0306419018818551

Putnam, R., (2000) Bowling Alone: The collapse and revival of American community, Simon \& Schuster, New York. 